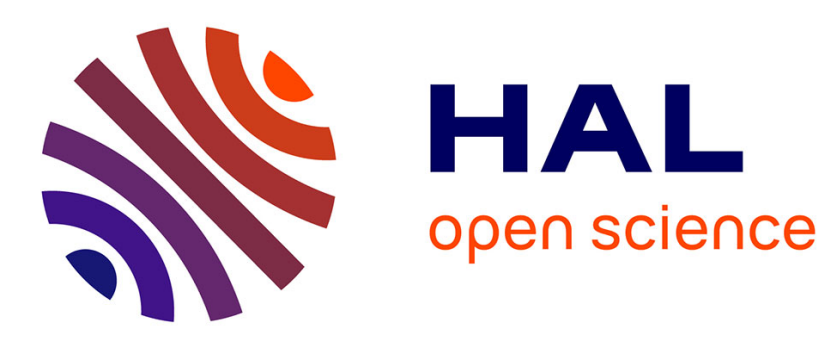

\title{
Blade-coating of yield stress fluids
}

Mathilde Maillard, Cédric Mézière, Pascal Moucheront, Christophe Courrier, Philippe Coussot

\section{To cite this version:}

Mathilde Maillard, Cédric Mézière, Pascal Moucheront, Christophe Courrier, Philippe Coussot. Bladecoating of yield stress fluids. Journal of Non-Newtonian Fluid Mechanics, 2016, 237, pp.16 - 25. 10.1016/j.jnnfm.2016.10.002 . hal-01784864

\section{HAL Id: hal-01784864 \\ https://hal-enpc.archives-ouvertes.fr/hal-01784864}

Submitted on 3 May 2018

HAL is a multi-disciplinary open access archive for the deposit and dissemination of scientific research documents, whether they are published or not. The documents may come from teaching and research institutions in France or abroad, or from public or private research centers.
L'archive ouverte pluridisciplinaire $\mathbf{H A L}$, est destinée au dépôt et à la diffusion de documents scientifiques de niveau recherche, publiés ou non, émanant des établissements d'enseignement et de recherche français ou étrangers, des laboratoires publics ou privés. 


\title{
Blade-coating of yield stress fluids
}

\author{
M. Maillard, C. Mézière, P. Moucheront, C. Courrier, P. Coussot* \\ Université Paris-Est, Laboratoire Navier (ENPC-IFSTTAR-CNRS), Champs sur Marne, \\ France \\ * Corresponding author: philippe.coussot@ifsttar.fr, +33181668544
}

\begin{abstract}
Blade-coating of pastes (yield stress fluids) consists of coating the fluid by pushing and spreading it over a solid substrate. It is a widely used process in civil engineering (mortars, cement pastes, rendering, paints) and in cosmetics (coating of gels or creams). Here we study this process experimentally by displacing horizontally a vertical plate partially immersed in the initially uniform layer of model yield stress fluid (Carbopol gel). We look at the impact of the initial fluid thickness, the scratched layer thickness, the fluid yield stress, and the blade velocity. The analysis is completed by rough PIV measurements. We show that the displacement of the blade at a constant distance from the solid plane induces, behind the blade, the formation of a uniform layer of thickness equal to 1.09 times this distance. In front of the blade the fluid forms a heap of growing volume. It appears that this heap advances over the initial uniform layer which induces a shear in a band between two almost undeformed blocks. It is shown that the shape of this heap results from the momentum balance on the fluid volume submitted to gravity and shear stress (along the shear-band). Nevertheless the force to apply on the blade is larger than the stress along the band times the band surface by a factor larger than 1.5, which might result from normal force due to heap weight and elastic deformation in the upstream static layer.
\end{abstract}

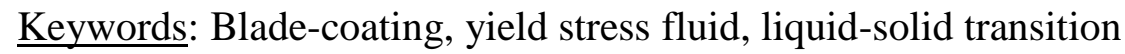

\section{Introduction}

In our everyday life we commonly extract objects from baths of fluids which are then spread over some solid surface (i.e. the substrate). In cosmetics we use our hands to extract cream, gel or foam from a container and then coat it on our skin. In cooking, often with the help of a knife, we coat melt chocolate, peanut butter or sauce on bread. In civil engineering we coat paint on walls, mortar between bricks, rendering over walls, cement paste on some ground, etc, with the help of a brush or a trowel. All these applications deal with yield stress fluids initially coated in the form of a thick layer over the substrate. This layer is then spread by moving the tool parallel to the substrate and maintaining the tool tip at a distance from the substrate, smaller than the initial layer thickness. Thus the flow results from the displacement, parallel to the bottom, of a more or less inclined blade plunged in a layer of yield stress fluid.

In general, in such a situation one aims at getting a uniform layer of a given thickness and minimizing the energy needed for that. However in all the above mentioned applications one can only rely on an empirical know-how to try to reach these objectives. The goal of the present work is to provide some basic fundamental knowledge of this process. 
This process is reminiscent of the blade-coating of liquids, which is one of the various possibilities to coat solid surface with a fluid [1]. In that case, some liquid layer is first coated onto a solid surface moving out of a bath of liquid and then a blade, positioned at some distance from the solid surface, tends to reduce the thickness of the liquid layer. In other cases, there is no initial coating stage, the bath of liquid is directly in front of the blade, which governs the flow characteristics and in particular the layer thickness remaining behind. Various industrial processes (food, textile or paper industries, civil engineering) rely on this technique to coat surfaces. The stakes of research in that field are to understand the fluid flow properties in order to optimize process parameters (geometrical characteristics of the blade, relative velocity of the blade and the surface to coat, rheological properties of the fluid) and to ensure the uniformity and the stability of the coated layer.
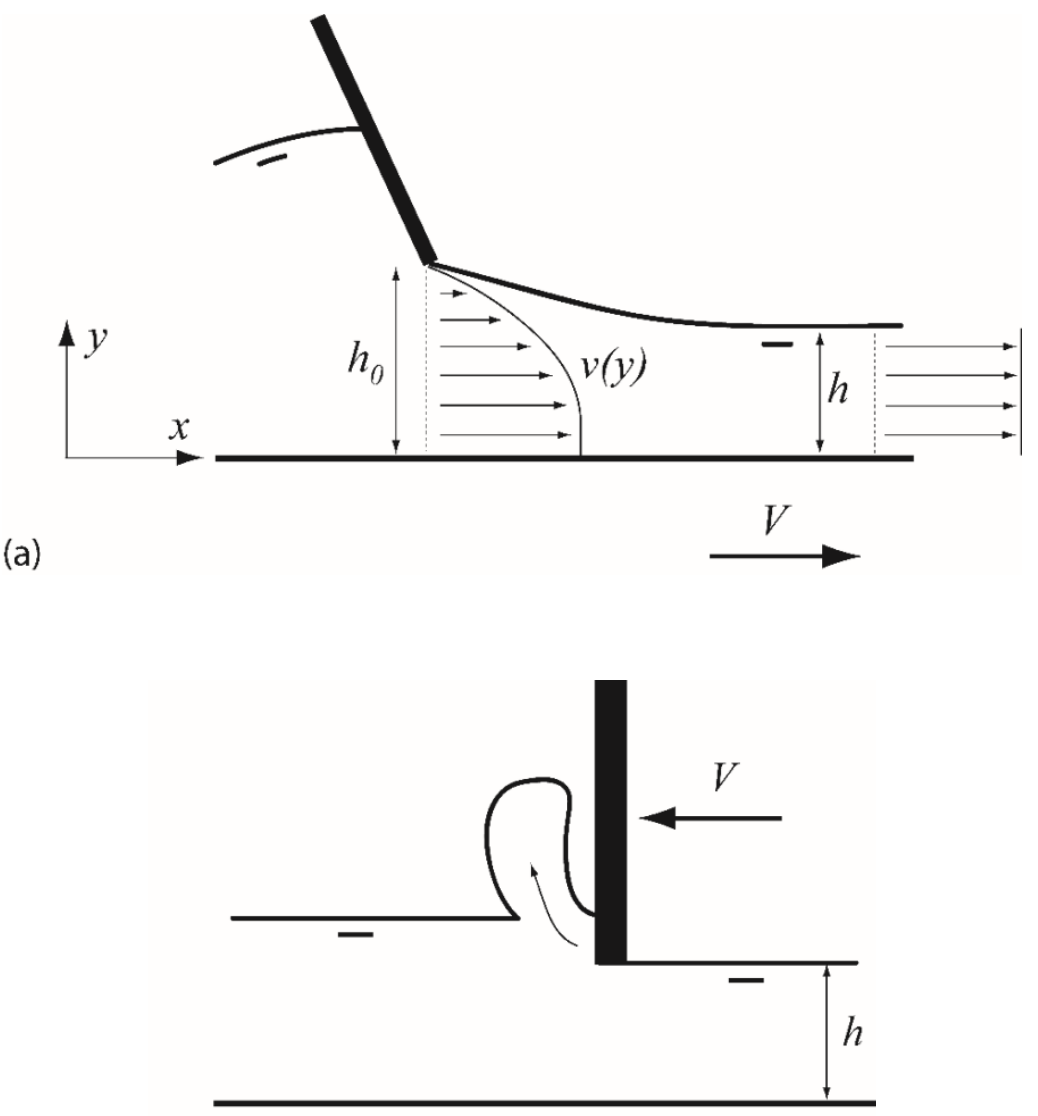

(b)

Figure 1: Schemes of the principle of blade-coating for fluids (a) and scratch test for solids (b).

Here we will focus on the simplest case of a solid substrate moving at a constant velocity $V$ and bringing with it some fluid amount (see Figure 1a). Thus we do not consider the case of a fluid reaching the blade with a velocity larger than that of the substrate. A blade is positioned at some place, which tends to retain some part of the fluid. Note that in the frame attached to 
the plane these conditions correspond to the coating process of yield stress fluids in cooking, cosmetics or civil engineering (see above). Let us now consider the flow characteristics in the frame attached to the blade. Assuming no wall slip along the solid surfaces, the velocity profile between the blade tip and the solid plane must respect two boundary conditions: $v(y=0)=V$ and $v\left(y=h_{0}\right)=0$. Since here the fluid approaches the blade essentially as a plug flow (i.e. $v(y)=V$ ) the velocity profile in the fluid below the blade is a "perturbation" of this profile (see Figure 1). To be more precise we define this perturbation as a scalar $p$ equal to the relative difference of the velocity integral and that of the uniform profile: $p=1-\int_{0}^{h_{0}}\left(v(y) / h_{0} V\right) d y$. Far from the blade the fluid is necessarily at rest relatively to the substrate and we assume it exhibits a uniform thickness $h$. The mass conservation then implies that $\int_{0}^{h_{0}} v(y) d y=h V$, so that we have $h=(1-p) h_{0}$.

For a homogeneous simple shear between the two boundaries, i.e. $v(y)=\left(1-y / h_{0}\right) V$, the perturbation is equal to $1 / 2$ so that we have $h=h_{0} / 2$. Actually, such a simple shear can only be obtained if the blade is replaced by a long horizontal solid plane in contact with the fluid and parallel to the substrate. Indeed a relatively thin blade essentially induces a local perturbation of the flow of the fluid layer lying on the substrate, and in that case the shear rate will be larger than for a homogeneous shear at the approach of blade tip and smaller at the approach of the substrate which tends to fix the fluid on it over a much larger distance than the blade thickness. In fact the exact response is governed by the constitutive equation of the material and the stress distribution. As a consequence we can expect a smaller perturbation for a shear-thinning fluid, and in particular for a yield stress fluid (the sheared region being more localized close to the blade tip), which leads to a value for $h$ higher than $h_{0} / 2$, but anyway smaller than $h_{0}$. As a consequence, even if we can hardly provide a full demonstration which would likely require a full set of numerical simulations, it seems reasonable to consider that the maximum perturbation corresponds to a simple shear, which means that $h=h_{0} / 2$ is the minimum value of the coated thickness that can be obtained. On the other hand, following the above reasoning we expect that the maximum value of the coated thickness to be $h_{0}$, a situation corresponding typically to a pure plastic flow for which the shear would be localized in an infinitely thin layer along the blade tip (see below).

More sophisticated existing studies dedicated to blade-coating tend to confirm these conclusions and go farther: for simple liquids $h$ remains inferior to the gap and larger than half its value [2], $h$ essentially depends on the blade geometry [3-5], $h$ increases with the blade inclination and slightly decreases with the blade velocity [5], shear-thinning effects increases $h$ which remains smaller than the gap [3-4, 6-7], elastic effects are in competition with viscous ones, decreasing $h[3,4,8]$. Some of these works present flow visualizations through streamlines [4-6] or qualitative direct observations [9]. It appears [9] that the flow shape and the volume of fluid at stake are strongly dependent on the fluid viscoelastic properties, leading to the formation of dead zones or even reverse flows. To our knowledge, only a very small number of works focused on coating flows with yield stress fluids: dip- 
coating [10-11], roll-over-web coating [12], coating with a (vertically free) blade moving along a layer with a focus on the flow instability [13], spreading of emulsions on glass plate with a special focus on wetting process and phase inversion at low thickness [14]. Recently a work focused on the flow through a wedge, induced by the displacement of the bottom plane [15]. In that case the whole fluid volume is initially inside the wedge and starts to flow at the beginning of the test, a situation which differs from that studied here for which the fluid volume pushed by the blade continuously grows. However, in this work, direct visualization through a wall provided interesting information on the internal flow characteristics of a yield stress fluid flowing through a wedge, a situation corresponding to that encountered in our case in the fluid region below the blade.

Considering now essentially the plastic behavior of the yield stress fluid, which is equivalent to have a strong shear-thinning behavior (see above), the perturbation should be extremely small and we can expect that $h \rightarrow h_{0}$. Under these conditions the blade-coating process for yield stress fluid is reminiscent of the scratch test used for solids [16]. In that case the material which has just been scratched tends to accumulate in front of the blade keeping its new shape (see Figure 1b). The obtained value for the thickness of the layer behind the blade is generally not discussed, it seems to be expected to be equal to $h_{0}$. In that frame the critical trend which is observed and discussed is the force exerted onto the blade by the material as a function of the material properties, the blade geometry and the depth of penetration [16].

Here we study experimentally the horizontal coating of simple yield stress fluids on a flat surface with a thin blade. We look at the overall aspects of the flow and the variation of the coated thickness as a function of the velocity, the fluid behavior and the distance between the blade and plane. First, we present the material, equipment and procedures (section 2). Then we discuss the general shape of the flow, focusing on the geometrical characteristics of the heap of fluid that forms ahead of the blade and on the force applied on the tool. We also look at the local velocity field inside the fluid through rough PIV measurements (section 3). Finally, we analyze and put in perspective our different results to suggest different models explaining the phenomena (section 4).

\section{Materials and methods}

\subsection{Materials}

As yield stress fluids, we used solutions of Carbopol 980 in water at different concentrations (see [17] for details on material preparation). To characterize their flow curves, rheometrical tests were carried out with a Bohlin C-VOR rheometer equipped with two circular rough parallel plates to prevent slippage (diameter $4 \mathrm{~cm}$; waterproof sandpaper of average particle diameter $120 \mu \mathrm{m})$. Sweep tests were performed: the stress was increased logarithmically in time (for $3 \mathrm{~min}$ ) and then decreased for the same time. The data in the decreasing ramp are considered as describing the material behaviour in steady state. These materials behave as simple yield stress fluids with negligible thixotropy. The flow curve representing the steady 
state shear stress as a function of the shear rate can be very well fitted over four decades of shear rates $\left[10^{-2} ; 10^{2} \mathrm{~s}^{-1}\right]$ by a Herschel-Bulkley model (see Figure 2) The resulting behavior in simple shear is $\tau<\tau_{c} \Rightarrow \dot{\gamma}=0$ (solid regime) and $\tau>\tau_{c} \Rightarrow \tau=\tau_{c}+k \dot{\gamma}^{n} \quad$ (liquid regime), where $\dot{\gamma}$ is the shear rate and $\tau_{c}, k$ and $n$ are parameters depending on the material characteristics. For our experiments, we prepared three Carbopol gels for which $\tau_{c}$ is 42,49 and $98 \mathrm{~Pa}$. In the following we will refer to the different materials through their yield stress value.

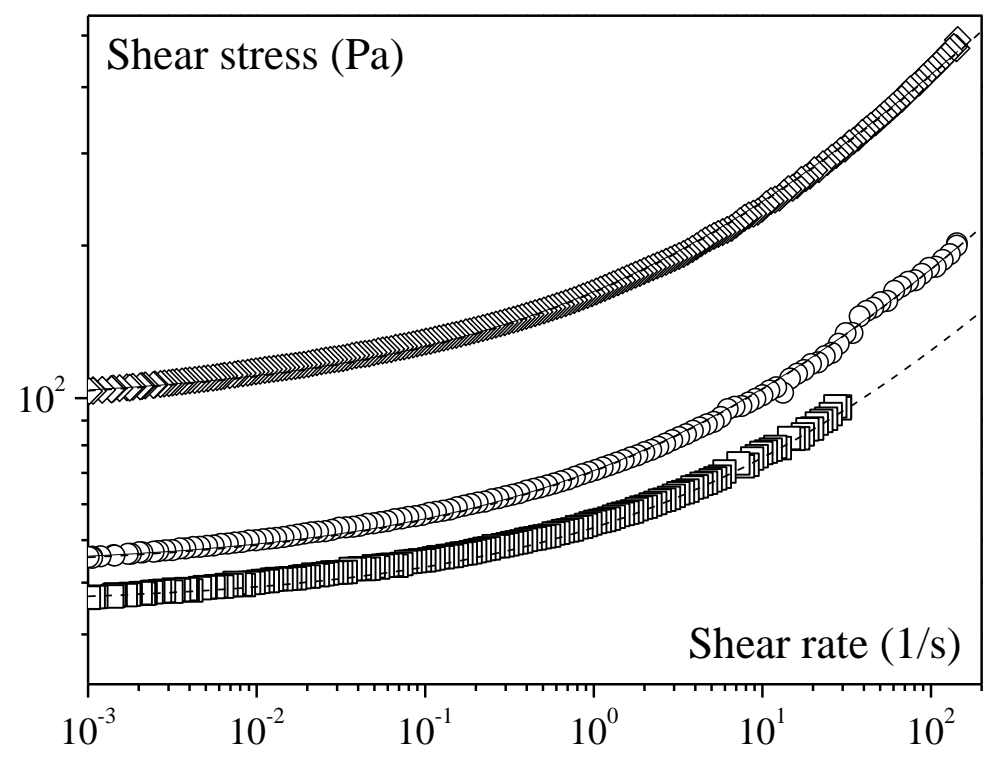

Figure 2: Flow curves of our Carbopol gels. The dashed lines correspond to a HerschelBulkley model fitted to data with $n=0.35$ and: $\tau_{c}=42 \mathrm{~Pa}, k=16 \mathrm{~Pa} . \mathrm{s}^{\mathrm{n}}$ (squares); $\tau_{c}=49$ $\mathrm{Pa}, k=24.5 \mathrm{~Pa} . \mathrm{s}^{\mathrm{n}}$ (circles) and $\tau_{c}=98 \mathrm{~Pa}, k=58 \mathrm{~Pa} . \mathrm{s}^{\mathrm{n}}$ (diamond).

\subsection{Blade-coating experiment}

\section{Set-up}

The set-up is composed of a parallelepiped Plexiglas channel put on a linear motor stage, a vertical blade, two force sensors, an acquisition system and a camera (see Figure 3). The channel is $140 \mathrm{~cm}$-long and $12 \mathrm{~cm}$-large. There are two $14.5 \mathrm{~cm}$-high lateral walls. The two extremities of the channel are free to prevent them from disturbing the flow. The channel may be moved over $80 \mathrm{~cm}$ at a velocity between 1 and $500 \mathrm{~mm} / \mathrm{s}$. The blade is a $12 \mathrm{~cm}$ side square and $2 \mathrm{~mm}$ thick piece of aluminum. This blade can be considered as perfectly rigid during our tests: under the action of a $10 \mathrm{~N}$ force at its extremity the induced displacement is of the order of $0.5 \mathrm{~mm}$, which is far smaller than the relative displacements of the blade and the channel observed in our tests. There is a $1 \mathrm{~mm}$ gap between the blade sides and the channel sides in order to limit the effects of wall frictions. Direct observations suggest that the fluid flow through this gap is negligible. A CCD camera is fixed to the structure that holds the blade and takes videos on the aspect of the flow around the blade seen from a channel side. The blade is 
directly linked to two $50 \mathrm{~N} \pm 10^{-2} \mathrm{~N}$ Utilcell double bending beam load cells. The structure of these sensors is such that we directly record the horizontal and vertical forces applied to the blade even if the point of application of this force is at some distance from the cell. Since force data presented here correspond to a vertical blade, these two forces are respectively the normal and tangential force applied to the blade. We also carried various experiments at different angles $\left(22^{\circ}, 45^{\circ}\right)$ of the blade around the blade tip and with regards to the vertical (positive angle in the flow direction) but in that case we only measured the thickness of the coated layer. Note that there is no wall slip of Carbopol gels along the aluminium plate. We checked that by putting a sample heap on the plate and inclining it very progressively. We could observe that the contact line situated upstream remained fixed even when the material started to flow and spread significantly over the plate.

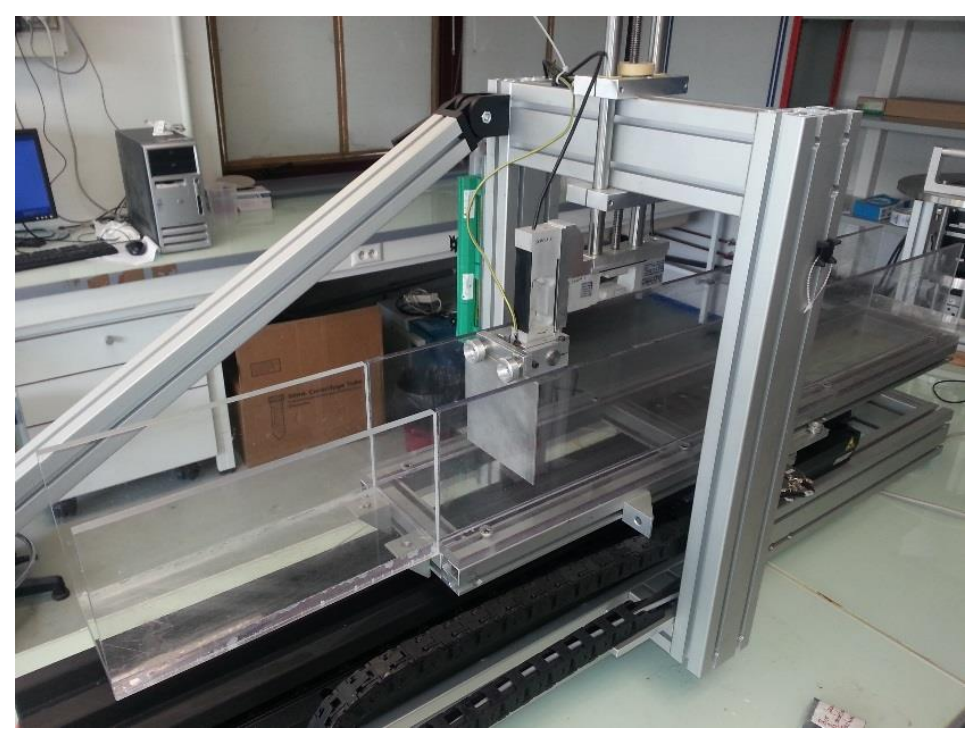

Figure 3: Overal view of the set up (camera not shown).

\section{Procedure}

In order to start each test with a uniform layer, we first pour a given volume of fluid, then the blade is plunged down to a certain depth inside this layer and the channel is moved horizontally so that the blade reaches the edge of the fluid layer. This gives the initial configuration, in the form of a 120-mm-wide layer, approximately $800 \mathrm{~mm} \mathrm{long}$, and of uniform thickness $h_{1}$. Afterwards the blade is removed and cleaned, and the channel is put back to its initial position. The blade is then moved down to a new distance $h_{0}$ from the channel bottom. The experiment is launched when the channel starts moving at a velocity $V$. It stops when the height of the heap of fluid that forms in front of the blade starts decreasing, which means that the blade is too close to the edge of the initial layer so that the heap starts to spread beyond this edge. Note that since in the final state of this experiment, a new layer of uniform thickness has formed, this situation can be used as the initial state of the next test. In our tests $h_{1}$ was varied between 5 and $37 \mathrm{~mm}$ and $h_{0}$ between 2 and $30 \mathrm{~mm}$ so as to scrape a layer of fluid of height $h_{1}-h_{0}$ equal to 3,5 or $10 \mathrm{~mm}$. Finally the experimental conditions for a given test are the set of parameters $\left(\tau_{c}, h_{1}, h_{0}, V\right)$ (see Table 1$)$. In each case the tested 
channel velocities were 1, 5, 50 and $100 \mathrm{~mm} . \mathrm{s}^{-1}$. All the tests shown in Table 1 for $98 \mathrm{~Pa}$ have also been carried out for an angle of the blade of $22^{\circ}$ and for an angle of $45^{\circ}$ relatively to the vertical direction.

\begin{tabular}{|c|c|c|c|c|c|c|c|c|}
\hline$\tau_{c}(\mathrm{~Pa})$ & $h_{1}(\mathrm{~mm})$ & $h_{0}(\mathrm{~mm})$ & $\tau_{c}(\mathrm{~Pa})$ & $h_{1}(\mathrm{~mm})$ & $h_{0}(\mathrm{~mm})$ & $\tau_{c}(\mathrm{~Pa})$ & $h_{1}(\mathrm{~mm})$ & $h_{0}(\mathrm{~mm})$ \\
\hline 98 & 37 & 27 & 49 & 17 & 7 & 42 & 17 & 7 \\
\hline 98 & 17 & 7 & 49 & 20 & 15 & 42 & 20 & 15 \\
\hline 98 & 30 & 25 & 49 & 15 & 10 & 42 & 15 & 10 \\
\hline 98 & 20 & 15 & 49 & 7 & 2 & 42 & 7 & 2 \\
\hline 98 & 15 & 10 & 49 & 5 & 2 & 42 & 5 & 2 \\
\hline 98 & 7 & 2 & & & & & & \\
\hline 98 & 5 & 2 & & & & & & \\
\hline
\end{tabular}

Table 1: Blade-coating test parameters

The recorded information is the horizontal position $d$ of the blade along the channel (initial state: $d=0$ ), and the normal (horizontal) $F_{x}$ and tangential (vertical) $F_{y}$ forces applied onto the blade all along this path. In addition, the geometrical characteristics of the flow are analyzed from the movies. Images are selected for each $5 \mathrm{~cm}$-displacement of the channel and different measures are taken directly on these pictures. The uncertainty on such measurements is about $0.5 \mathrm{~mm}$ in the uniform regions (see below) but may reach a few millimeters in more complex regions.

\subsection{Description of internal flow characteristics}

We determined experimentally the velocity field inside the fluid using the Particle Image Velocimetry technique. Due to technical limitations, we could not perform a full proper analysis using a laser sheet and small tracers. Indeed the free surface of the heap largely deforms during the experiment while not being perfectly planar; thus it strongly diffracts and reflects the laser light, preventing from detecting the tracers. Instead, we used the bubbles inside the fluid to obtain approximate velocity fields.

The films were analyzed using the commercial software DaVis, with the protocol described in $[10,17]$. Each image was divided into squares of correlation of 24 x 24 pixels (35 pixels $=1$ $\mathrm{cm})$. On a given stack of images, we used a cross-correlation technique comparing successive images through the determination of the average particles displacements between two pictures in every correlation square. The first image was compared to the second one, the second one to the third one and so on, taking into account all the images of a given stack. Then all the correlations were averaged to give the final velocity field of the stack of images. In these fields, there is one velocity per correlation square. 
The flow fields were obtained by averaging the pictures taken during about a $5 \mathrm{~cm}$ displacement of the channel. Thus, the number of images per stack used to compute an averaged velocity field varies for each test and decreases with the plate velocity. Using a constant displacement to compute the velocity field in the fluid whatever the plate velocity allows an easier comparison between the data. When the blade is at a distance smaller than 10 $\mathrm{mm}$ from the bottom of the channel, the thickness of the fluid region is too small to compute a proper velocity field in this area.

\section{Results}

3.1 General shape of the flow
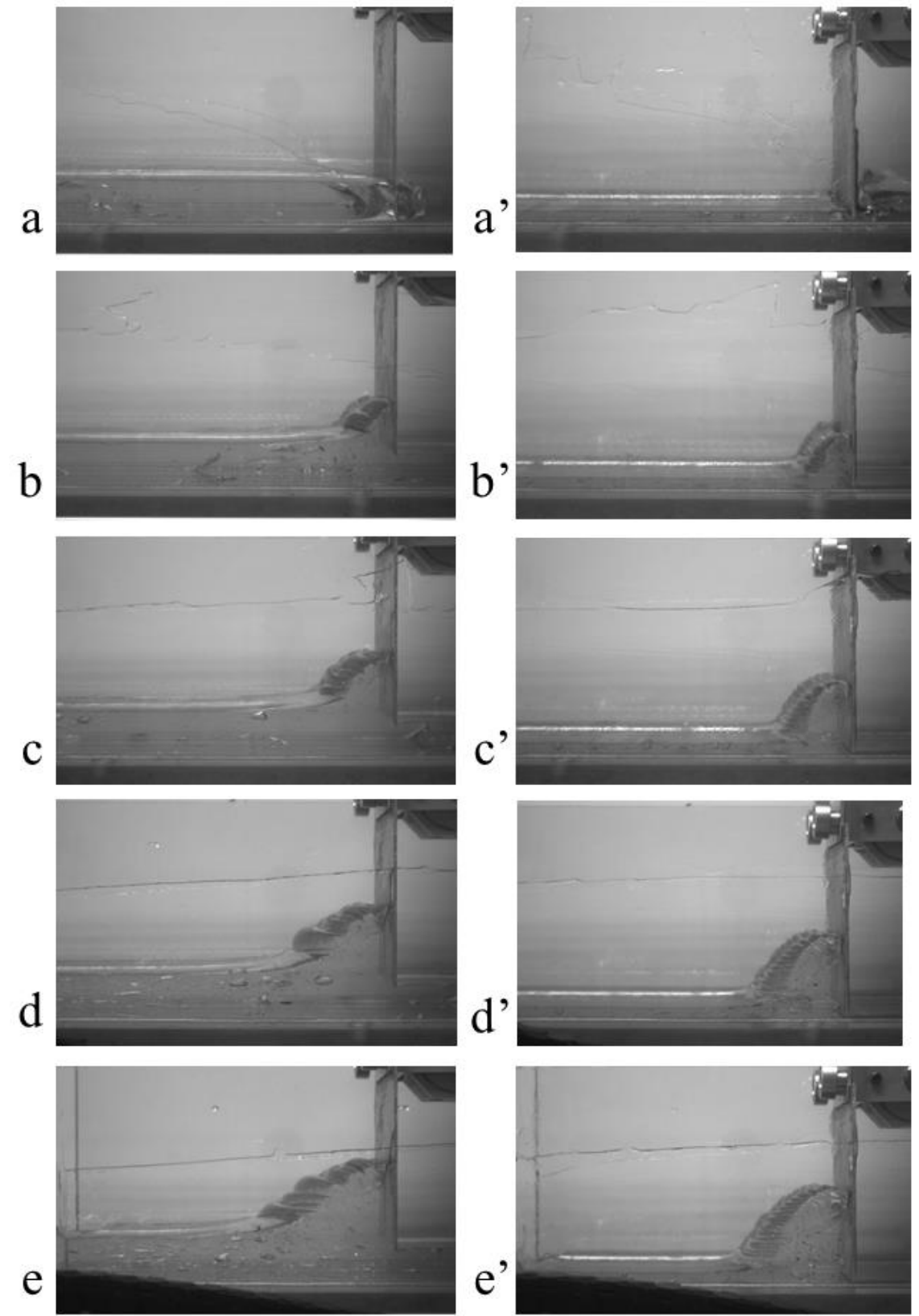

Figure 4: Aspect of the fluid at different times during the blade displacement (at a velocity $V=5 \mathrm{~mm} / \mathrm{s})$ over an initially uniform layer of yield stress fluid $\left(\tau_{c}=98 \mathrm{~Pa}\right)$ : (left) 
$h_{1}=25 \mathrm{~mm}$ and $h_{0}=20 \mathrm{~mm}$, (right) $h_{1}=7 \mathrm{~mm}$ and $h_{0}=2 \mathrm{~mm}$, after different distances $d$ of displacement of the blade : (from top to bottom) 0, 150, 300, 450, $600 \mathrm{~mm}$.

As the blade is moved along the fluid layer two regions can be distinguished (see Figure 4): behind the blade the fluid layer keeps a thickness close to $h_{0}$; ahead of the blade the fluid is strongly deformed, a heap of increasing volume develops. Note that except in a $1 \mathrm{~cm}$ wide area close to the channel walls, there is no observable transversal flow in the heap of fluid. The thicknesses of the fluid layers ahead and behind the blade are approximately uniform along the channel width. Note that the photos are misleading concerning this point. They were taken so as to have a " $3 \mathrm{D}$ " view of the fluid shape, and thus they give some idea of the fluid thickness along the $3^{\text {rd }}$ dimension, i.e. along the channel width, but the view in that direction is compressed and due to the perspective the $12 \mathrm{~cm}$ of the channel width appear as a few centimeters on the photo. In fact direct measurements at different moments during the experiments made it possible to check that a given distance from the blade the depth slightly varies over a distance of the order of $5 \mathrm{~mm}$ (about $2 \mathrm{~mm}$ behind the blade) from the wall, while the depth is uniform along the rest of the width. This supports the conclusion that the flow characteristics are identical over more than $90 \%$ of the width, and we can consider it as a "2D" flow. We can also observe ondulations along the free surface of the heap. This is likely due to some instability in the process of accumulation of material behind the blade. It is reminiscent of the instability (coiling) occurring when a filament of yield stress fluid falls over a solid surface [18], but we have no further explanation at this stage.

\subsection{Geometrical characteristics of the flow}

After about a 1cm-displacement of the blade, the fluid layer coated behind the blade reaches a constant shape: it increases progressively from $h_{0}$ to $h$ over a distance of a few millimeters and is uniform beyond this distance. In Figure 5 we plot $h$ vs $h_{0}$ for all the tests at different velocities, yield stresses, layer thicknesses, blade angles and gaps. In this representation all the data fall along a master curve, which can be well represented by:

$h=1.09 h_{0}$ 


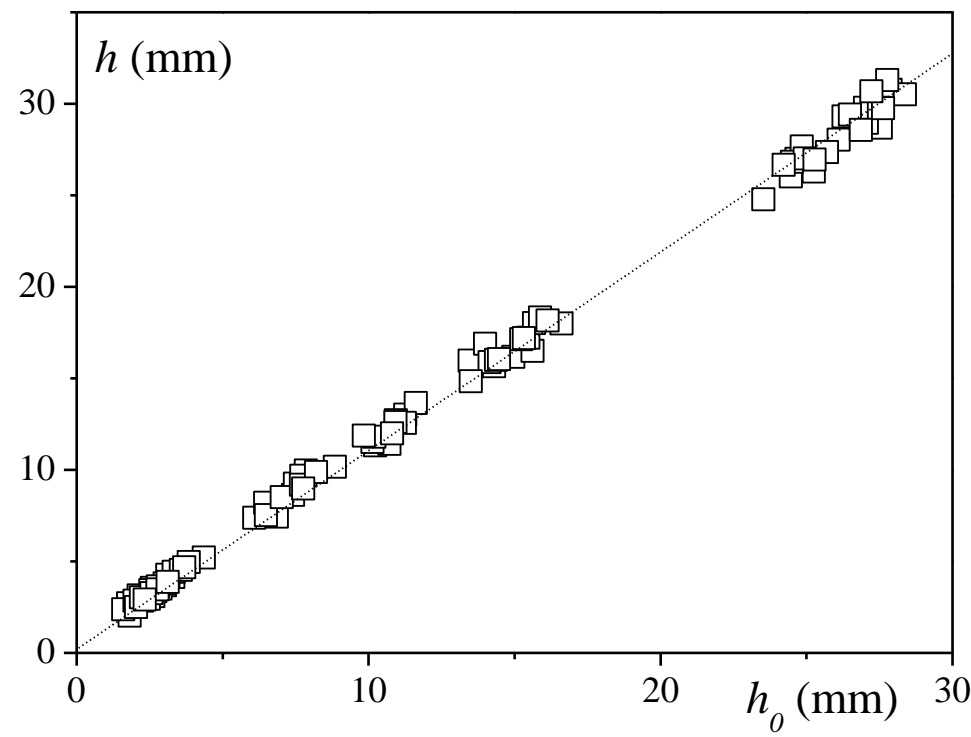

Figure 5: - Uniform coated layer thicknesses as a function of the gap between the blade and the plane for all the tests. The dotted line corresponds to equation (1).

During a first stage the shape of the free surface in front of the blade in a vertical plane along the channel axis is not simple and evolves as the blade progresses. We did not study further this stage during which surface tension and deformations in the solid regime might play a major role. Beyond some length of relative displacement we can observe a well-developed heap (see Figure 6) which, as a first approximation, keeps an almost constant shape (of varying scale) that we can describe with the help of only two characteristic lengths: its maximum height $H$ and its longitudinal extension $L$ ahead of the blade (see Figure 6). The different lengths $(H, L, h)$ are measured on the pictures, all along the channel displacement. The uncertainty on $H$ measurements is about $2 \mathrm{~mm}$, due to the irregularity of the free surface of the heap, while it is around $10 \mathrm{~mm}$ on $L$ due to the difficulty to identify precisely the end of the heap. 


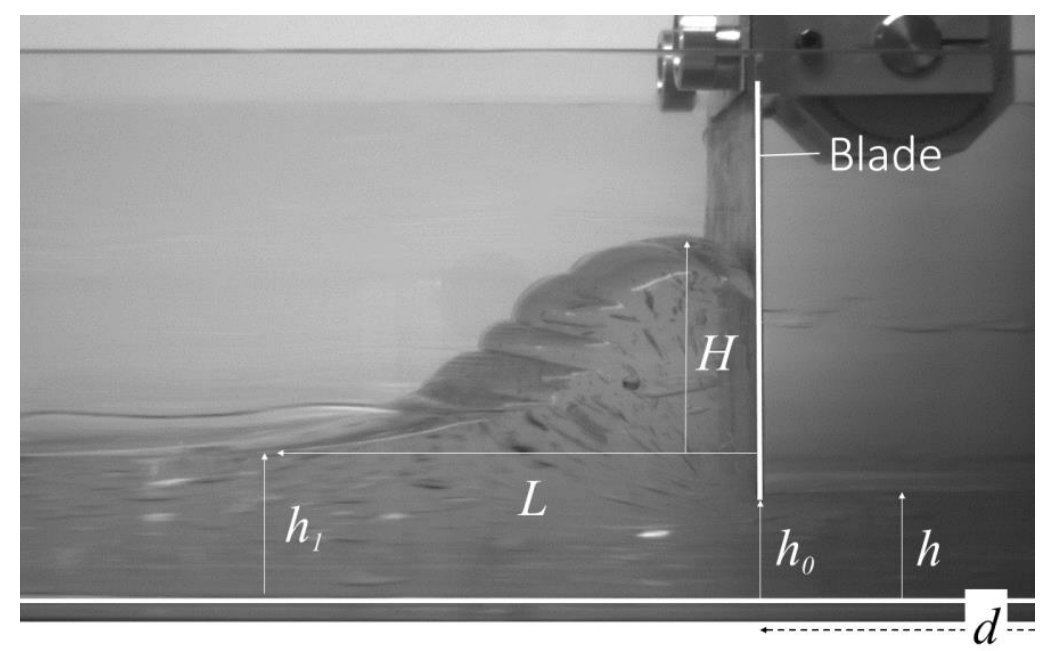

Figure 6: Notations. The picture here corresponds to the flow of the fluid with a yield stress $\tau_{c}=49 \mathrm{~Pa}$, at a blade velocity $V=50 \mathrm{~mm} / \mathrm{s}$, an initial layer thickness $h_{1}=10 \mathrm{~mm}$ and a gap $h_{0}=5 \mathrm{~mm}$.

Typical examples of the growth of the height and length of the heap as a function of the displacement of the channel are shown in Figure 7. It appears that in all cases $L$ and $H$ increase with the distance, but their rate of increase decreases progressively as the channel advances. Moreover, as it appears in Figure 7, the aspect ratio of the heap (i.e. $H / L$ ) can evolve in a very different way for two tests even for the same scratched layer thickness $(5 \mathrm{~mm}$ in those cases): the length is significantly larger than the height when $h_{0}$ is relatively large, say $h_{0} \geq 7 \mathrm{~mm}$, while otherwise $\left(h_{0}<7 \mathrm{~mm}\right.$ ) the length and height of the heap remain of the same order. Note that the distinction of these regimes, and the identification of an approximate threshold in terms of $h_{0}$, will be confirmed by the other measurements presented later. Obviously it would be preferable to quantify the transition between these two regimes in terms of a dimensionless number. We could for example think of a criterion based on the aspect ratio $r=\left(h_{1}-h_{0}\right) / h_{0}$. However this cannot be sufficient here because the other characteristic lengths of the flow, i.e. the height and length of the heap, continuously evolve during the flow, which means for example that other aspect ratios might play a role. As a consequence here we will simply retain the above basic criterion of the transition, keeping in mind that we cannot say whether it is appropriate out of our specific range of tests.

Let us now look more precisely at the variations of $H$ as a function of $L$. It seems that there is a fast initial increase of $L$ up to $L_{0}$, which we were not able to measure. Then it appears (see Figure 8) that $H^{2}$ is an affine function of $L$ in our range of measurements, namely we have:

$$
H^{2}=\alpha\left(L-L_{0}\right) \quad\left(\text { for } L>L_{0}\right)
$$

We have no clear explanation about the origin of the constant $L_{0}$ which does not seem to vary in a simple way with the experimental conditions. It might depend on the initial exact 
conditions of penetration of the blade and the complex deformation during the initial stage. Obviously a complete modelling of the process from the very beginning of the flow should be able to include the initial point $L=0=H$. However, for a sufficiently long motion this initial variation becomes negligible. As a consequence in the following we will only consider the coefficient of variation $\alpha$ as the hallmark of the shape dynamics of the heap. It appears that $\alpha$ increases slowly with the velocity and is approximately proportional to the yield stress (see Figure 9).

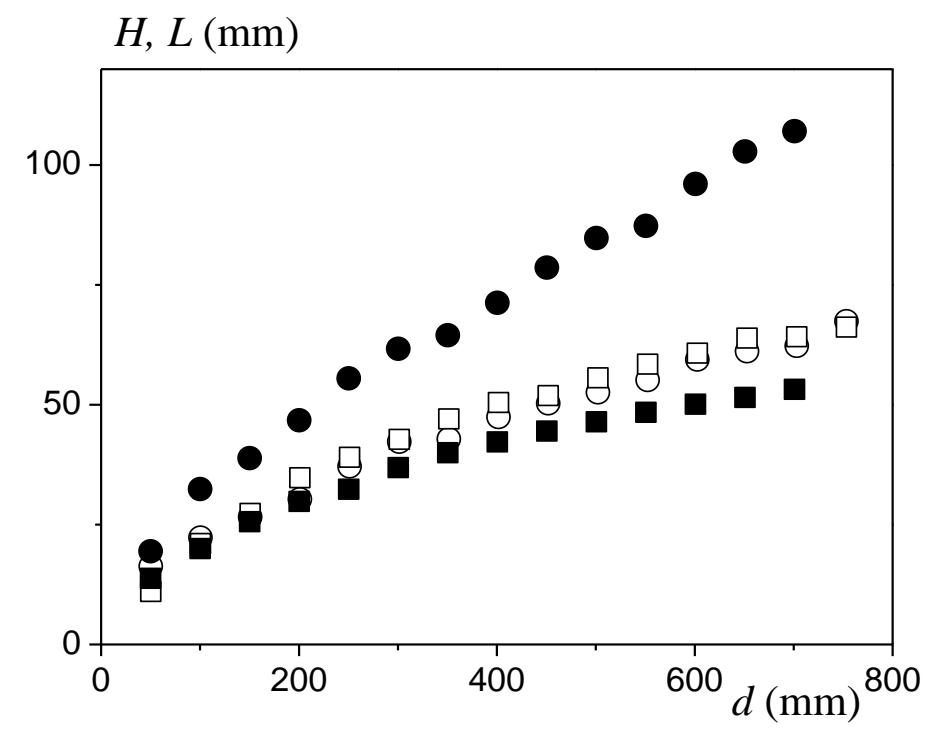

Figure 7: Height (squares) and length (circles) of the heap formed behind the blade as a function of the displacement for flows with $\tau_{c}=98 \mathrm{~Pa}$ and $V=1 \mathrm{~mm} / \mathrm{s}$, and: (filled symbols) $h_{1}=20 \mathrm{~mm}, h_{0}=15 \mathrm{~mm}$, or (open symbols) $h_{1}=7 \mathrm{~mm}, h_{0}=2 \mathrm{~mm}$.

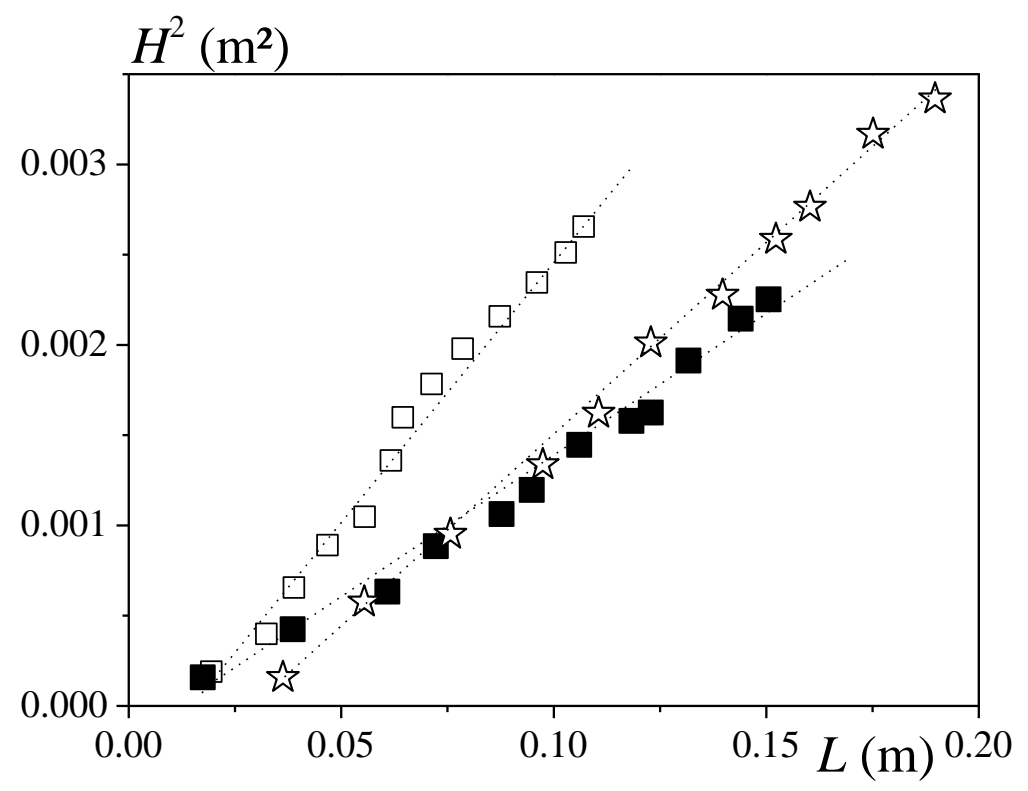


Figure 8: Squared height as a function of length of the heap formed behind the blade for flows with $\tau_{c}=98 \mathrm{~Pa}$ and $V=1 \mathrm{~mm} / \mathrm{s}$, and: (filled squares) $h_{1}=20 \mathrm{~mm}, h_{0}=15 \mathrm{~mm}$, (stars) $h_{1}=17 \mathrm{~mm}, h_{0}=10 \mathrm{~mm}$, and (open squares) $h_{1}=7 \mathrm{~mm}, h_{0}=2 \mathrm{~mm}$. Dotted straight lines are guides for the eye.

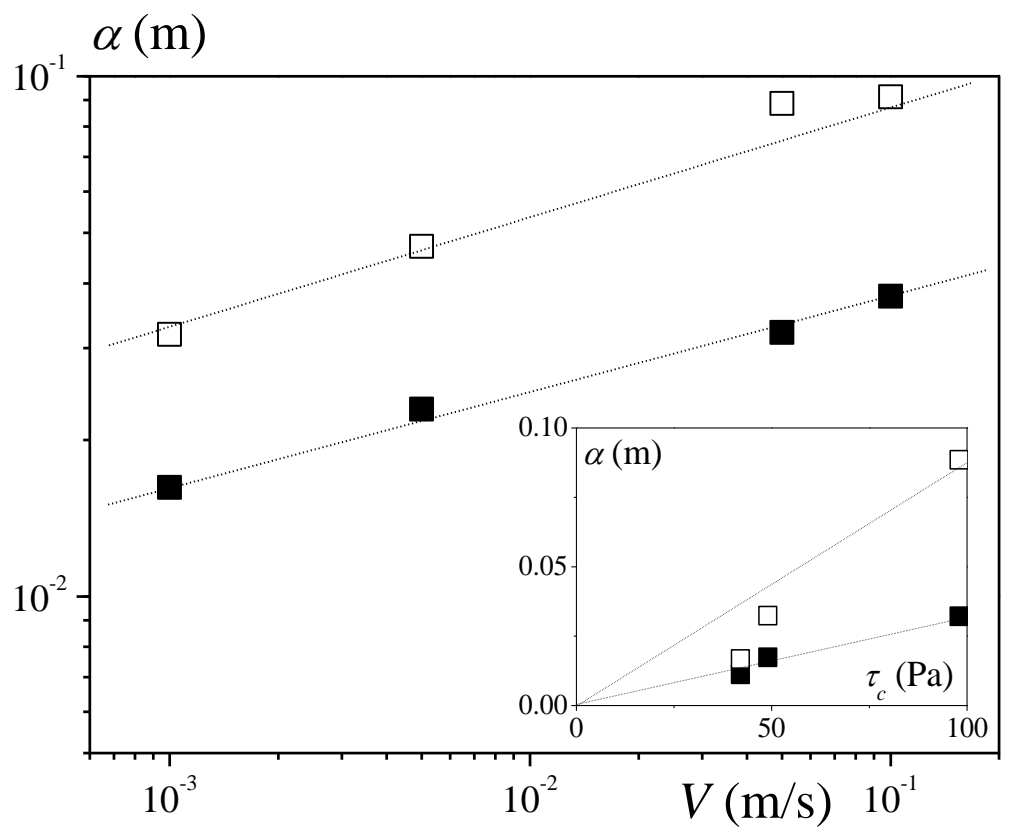

Figure 9: Values of the coefficient $\alpha$ (equation (2)) as a function of the blade velocity for $\tau_{\mathrm{c}}=98 \mathrm{~Pa}$ and: (filled squares) $h_{1}=20 \mathrm{~mm}, h_{0}=15 \mathrm{~mm}$ and (open squares) $h_{1}=7 \mathrm{~mm}$ , $h_{0}=2 \mathrm{~mm}$. The inset shows the variations of $\alpha$ as a function of the yield stress for $V=$ $50 \mathrm{~mm} / \mathrm{s}$ in these two situations.

\subsection{Forces}

For all tests the tangential force initially increases when the blade is displaced then it seems to reach a plateau (see Figure 10). On the contrary the normal force continuously increases during the displacement. In the following we will focus on the normal force, which will be referred to as the "force". Once again we apparently observe two regimes: for $h_{0} \geq 7 \mathrm{~mm}$ the force curves superimpose, which means that the force only depends on the scratched thickness $\left(h_{1}-h_{0}\right)$ (which is constant for all tests shown in Figure 10), and thus on the amount of fluid removed (see Figure 10); for $h_{0}<7 \mathrm{~mm}$ even for the same scratched value, the force is slightly lower (see Figure 10).

It appears that $F$ is proportional to $\sqrt{d}$ (see Figure 10):

$F=\beta \sqrt{d}$ 
where $\beta$ is a coefficient which depends on the experimental conditions. For $h_{0} \geq 7 \mathrm{~mm} \beta$ is constant for a given scratched thickness (we do not have enough data in the other regime to reach a conclusion on that point). This parameter is approximately proportional to the yield stress but varies very slowly with the blade velocity (see Figure 11). Moreover it finally seems to depend essentially on the value of $h_{1}-h_{0}$ and not so much on the regime (i.e. either $h_{0} \geq 7 \mathrm{~mm}$ or $h_{0}<7 \mathrm{~mm}$ ) (see Figure 11).

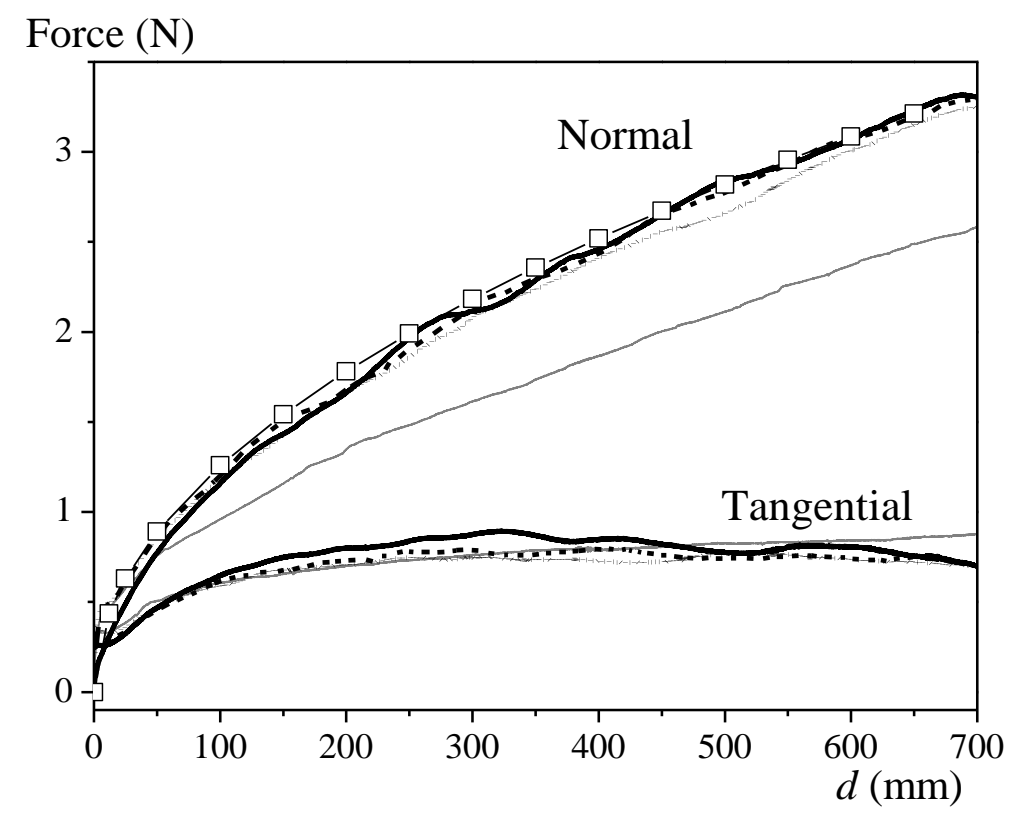

Figure 10: Tangential and normal forces as a function of the displacement for $V=5$ $\mathrm{mm} / \mathrm{s}, \tau_{c}=98 \mathrm{~Pa}$ and: (thick continuous line) $h_{1}=25 \mathrm{~mm}, h_{0}=20 \mathrm{~mm}$, (thick dash line) $h_{1}=20 \mathrm{~mm}, h_{0}=15 \mathrm{~mm}$, (thick dotted line) $h_{1}=15 \mathrm{~mm}, h_{0}=10 \mathrm{~mm}$, (thin line) $h_{1}=7 \mathrm{~mm}, h_{0}=2 \mathrm{~mm}$. The symbols correspond to equation (3) with $\beta=0.126 \mathrm{~N} \cdot \mathrm{mm}^{-1 / 2}$. 


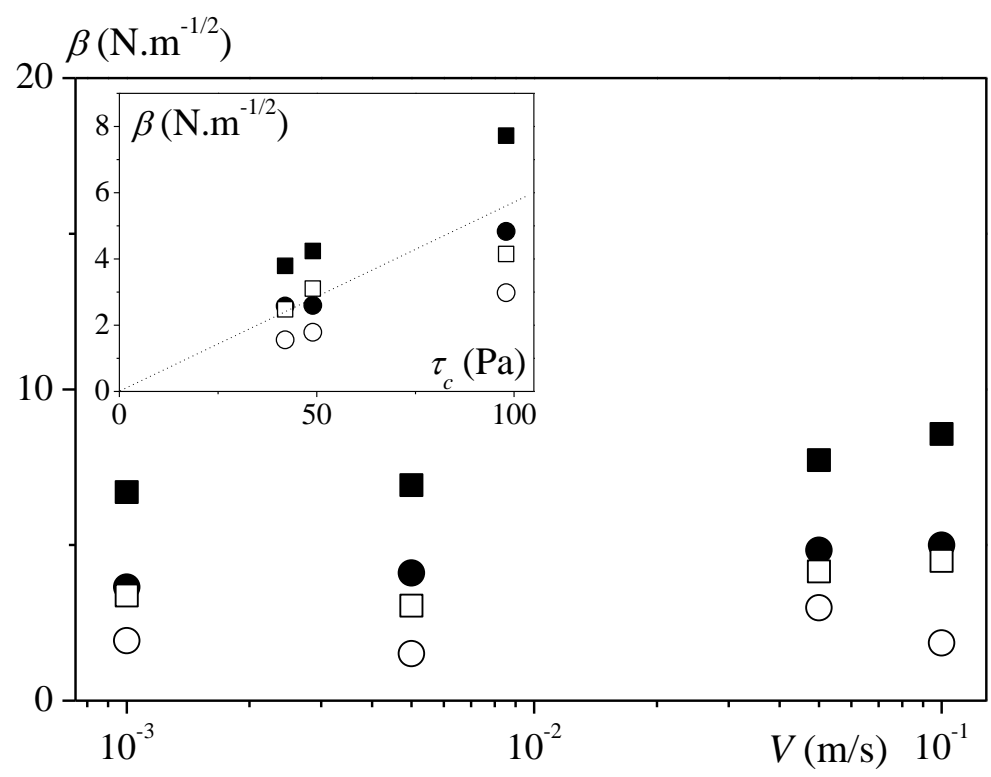

Figure 11: Values of the coefficient $\beta$ (equation (3)) as a function of the blade velocity for $\tau_{c}=98 \mathrm{~Pa}$ and: $h_{0}>7 \mathrm{~mm} \quad h_{1}-h_{0}=10 \mathrm{~mm}$ (filled squares) or $h_{1}-h_{0}=5 \mathrm{~mm}$ (filled circles); $h_{0}<7 \mathrm{~mm}$ and $h_{1}-h_{0}=5 \mathrm{~mm}$ (open squares) or $h_{1}-h_{0}=3 \mathrm{~mm}$ (open circles). The inset shows the variations of $\beta$ as a function of the yield stress for $V=50 \mathrm{~mm} / \mathrm{s}$.

\subsection{Visualization of the flow}

Our experimental determination by PIV of the velocity field can be considered as a rough view of reality as it relies on the bubbles as tracers and it takes 3D images in white light to compute 2D fields. Here we use it essentially to appreciate the different regions of flow in a typical case. Moreover, due to the uncertainty on data it is not relevant to attempt to extract precise information from a comparison of the results when varying the flow parameters. Recall that velocity fields are averaged over a time period corresponding to a $5 \mathrm{~cm}$ displacement of the plane. This displacement does not affect our (qualitative) analysis below. Indeed the corresponding maximum variation of $H$ or $L$ during that period is $20 \%$ (for the second picture of Figure 12), falling to $15 \%$ then $10 \%$ for the next pictures. Figure 12 shows a series of pictures of the velocity field in the frame of the blade at different steps of displacement of the plane. Remarkable features can already be extracted from this rough view:

- The uniform layers ahead of the heap and behind the blade are apparently at rest with regards to the channel;

- The heap in front of the blade has a velocity close to that of the blade, which suggests that it is moved as a block by the blade; 
- There is a region of transition between the heap and a layer almost at rest along the channel; overall the fluid is essentially sheared in this region of approximately uniform thickness $\delta$, i.e. this region ensures the transition from the heap moved almost as a block with the blade and the layer at rest on the channel.

Thus, the coating in a channel of a yield stress fluid with a vertical thin blade consists of two solid regions moving relatively to each other at a velocity $V$ thanks to an approximately uniformly sheared layer. The characteristic shear rate in this region is $V / \delta$. Since the angle between the shear-band direction and the horizontal is small, the length of this sheared layer is approximately $L$. This aspect of the velocity field seems close to that found for the flow through a wedge, i.e. with a large plug flow above a relatively thin sheared region [15].

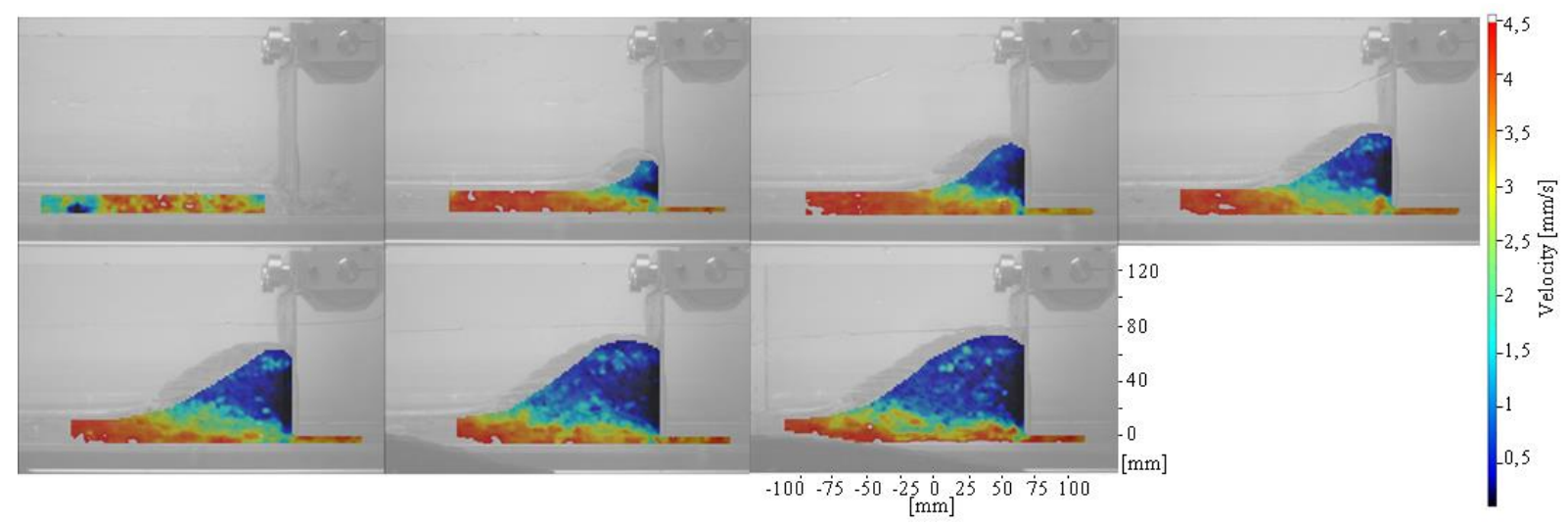

Figure 12: Evolution of the experimental horizontal velocity field during the bladecoating in a channel filled with yield stress fluid $\left(\tau_{c}=98 \mathrm{~Pa}, V=5 \mathrm{~mm} / \mathrm{s}, h_{1}=17 \mathrm{~mm}\right.$, $h_{0}=7 \mathrm{~mm}$ ). The pictures correspond to successive steps of displacement of $100 \mathrm{~mm}$. In this representation the blade has a velocity equal to zero (dark blue) while the channel has a velocity $V$ (red). The color bar is in $\mathrm{mm} / \mathrm{s}$.

\section{Discussion}

\subsection{General considerations}

Our observations suggest to see the blade-coating mainly as the displacement of a fluid amount (heap) above a layer of fluid at rest. During such a process most of the two parts of material remain in the solid regime but the fluid will be sheared in a relatively small layer at the interface between these two regions. The size of the heap grows in time as the blade advances, leading to an increase of the force needed to push the blade. In the following we analyze further the variations of the flow characteristics in the light of this scheme. At least two different regimes (with different evolutions of the force and heap geometry with the initial conditions) can be observed depending on the distance (gap) between the blade tip and the solid plane. Although we do not have sufficient data here to firmly support this conclusion the possible origin of the transition between the two regimes is that the thickness of the shearband becomes of the order or lower than the gap. 
The observation of a sheared layer of relatively small and almost constant thickness are reminiscent of the flow characteristics observed for the motion of a yield stress fluid through a large cylindrical pore [19] and the penetration [17] or withdrawal of a plate through a bath of yield stress fluid [10-11, 20]. In these cases it was found that the flow develops in the form of two rigid blocks of fluids sliding over each other with the help of an almost uniformly sheared layer of thickness varying slowly with the velocity.

Note that in this frame the vertical force is neglected. If the heap was growing as a result of an upward vertical flow we would expect the vertical force to continuously grow as the heap grows in size. Our observations (from videos) in fact show that the heap does not form in that way: the fluid (upstream) just reached by the wedge tip tends to be squeezed by the wedge advancement and it seems that some part of it tends to move and slowly climb the wedge up to the plate. This motion is in particular at the origin of the undulations which form along the free surface. Under these conditions the relative motion of the fluid wedge and the plate is not a simple upward motion, but may be closer to a progressive extension of the interface by the addition of new material, which likely explains the saturation of the vertical force.

\subsection{Coated thickness}

We observed (see Figure 5) that whatever the flow and materials parameters, in our range of tests, the coated thickness is larger than the gap by a factor of about $10 \%$ (equation (1)). This result differs from any of the result obtained so far with either Newtonian, non-Newtonian or solid materials (see Introduction) for which the coated thickness is smaller than the gap. More surprising, the result in the form of equation (1) is independent of all parameters of the flow: blade angle, velocity, gap, initial thickness, fluid yield stress.

This effect is reminiscent of the swelling of certain polymer solutions at their exit of a die, as a result of normal stress effects. There are however several reasons for which such effects cannot be at the origin of this swelling: normal stress effects generally appear at sufficiently large velocities whereas here we observe the same swelling in all our range of velocities (over 2 decades); when increasing the initial layer thickness, normal stress effects taking place in the sheared layer would concern a smaller fraction of the flow and would become smaller, whereas here we observe no impact of the initial layer thickness; even for viscoelastic liquids the coated layer has been observed to be smaller than the gap.

Another possibility is to have a velocity profile different from that shown in Figure 1a, now with a velocity larger than that of the channel in some region. If such an effect was sufficiently marked, the mass conservation would impose a larger thickness when the whole velocity profile gets back to the uniform channel velocity. However such an effect can only result from a sufficient fluid inertia. It can hardly be expected to occur at our lowest velocities and it should vary with the velocity.

The explanation we suggest relies on an aspect of the fluid behavior we have so far neglected, namely its elastic behavior in the solid regime. Indeed, according to our description of the process we have a mass of material (i.e. the heap) pushed by a blade and moving above a fluid layer essentially at rest. The heap of material transported by the blade along the channel may be seen as an object displaced over a layer of elastic solid material. As shown in [17] when 
some solid object is displace in a yield stress fluid elastic deformations in the solid regime take place in some region around a liquid region surrounding the object. Here we may thus have some elastic deformation in the solid regime in the fluid region remaining in its solid regime ahead and below the heap. Then, when the object is removed, i.e. after the blade motion beyond this region, these deformations may be recovered. Finally the amount of fluid behind the blade somehow behaves as a hand slightly pushed against a mattress and displaced along the surface of the mattress. Note however that this last argument is only a rough qualitative analogy which could hardly be further elaborated since a mattress is in general significantly compressible, which is not the case of our materials. Although this argument seems qualitatively sound we have no further argument to explain the proportionality between the gap size and the coated thickness.

\subsection{Heap geometry}

The shape of the heap formed in front of the blade mainly results from the balance between the force exerted on it from below and its tendency to stop flowing below the yield stress. Such a behavior is reminiscent of the deposits formed by stoppage of yield stress fluids over solid surfaces [21-22]. We can use similar arguments for quantitatively estimating the characteristics of such a flow. We describe the heap shape in 2D (in a vertical plane along the channel direction) through its height $(Y(x))$ above the sheared region, which is assumed to be horizontal. Then we assume that the pressure is hydrostatic and neglect the atmospheric pressure, i.e. $p=\rho g(Y-y)$, where $\rho$ is the fluid density and $g$ the gravity. Note that this assumption is known to be valid essentially for small slopes of the free surface, i.e. small values of $H / L$, which is not always the case in our tests and could be at the origin of a discrepancy between the theory and the data in some cases. Applying the momentum equation over a volume corresponding to a fluid layer between two vertical planes situated at $x$ and $x+d x$ we deduce, after integration of the pressure over the corresponding height, the following differential equation for the heap height:

$$
\rho g Y \frac{d Y}{d x}=-\tau
$$

where $\tau$ is the shear stress amplitude in the sheared layer, which is assumed to be uniform. We finally get after integration of (4) along the sheared layer:

$$
\rho g \frac{H^{2}}{2}=\tau L
$$

which may also be expressed

$$
\frac{H^{2}}{L}=\alpha=\frac{2 \tau}{\rho g}
$$

Owing to our observations we can consider that the stress results from the uniform simple shear of a layer of thickness $\delta$, so that we have

$\tau=\tau_{c}+k(V / \delta)^{n}$ 
which may be approximated by $\tau \approx \tau_{c}$ for sufficiently slow flows.

The expression (6) along with (7) predicts trends in agreement with our experimental observations: $\alpha$ is approximately proportional to $\tau_{c}$ and, if the variations of $\delta$ are negligible, slowly varies with the velocity. It also predicts that for a given scratched volume, i.e. $H L / 2 \approx$ Cst. , we will have the scaling laws $H \propto \tau_{c}^{1 / 3}$ and $L \propto \tau_{c}^{-1 / 3}$, which means that for increasing yield stress the heap will be higher and shorter, in agreement with our experimental observations.

Figure 13 gathers the experimental results for $\alpha$ represented as a function of a shear stress defined as in (7). In the latter expression we used $\delta=15 \mathrm{~mm}$ for $h_{0} \geq 7 \mathrm{~mm}$ and $\delta=10 \mathrm{~mm}$ for $h_{0}<7 \mathrm{~mm}$ which correspond to rough estimations from our PIV observations. In fact, since there are two terms in the stress expression and only one of them involves a power of $\delta$ at an exponent significantly smaller than 1 , the exact value for $\delta$ in a range of say [10-20 $\mathrm{mm}$ ] does not impact the conclusions below. For example in this range the maximum horizontal shift of data around those shown in Figure 13 is $+8 \%$ and $-5 \%$, inducing a possible variation of the slopes discussed below of $-8 \%$ and $+5 \%$.

For all data with $h_{0} \geq 7 \mathrm{~mm}$ we effectively find a single linear relationship as expected from (6), which confirms the general scaling obtained through the above theoretical approach. In that case the factor of proportionality is nevertheless slightly smaller than in theory, i.e. 1.7 instead of 2. We can finally consider that as long as the gap is larger than about $7 \mathrm{~mm}$ the heap behavior is rather well described by the above scheme and theory.

Linear relationships are also obtained for smaller gaps. However now different values for the coefficient of proportionality are obtained when the aspect ratio varies. Moreover this coefficient is now significantly larger than 2 (see Figure 13). This means that some of our assumptions in the above theory fail. The hydrostatic pressure distribution might now be invalid, which would directly lead to a coefficient different than 2 in (6). Another possibility is that the stress is in fact significantly larger than assumed (expression (7)), in particular at the approach of the blade tip, which would lead to a larger apparent coefficient when using (7) in (6). 


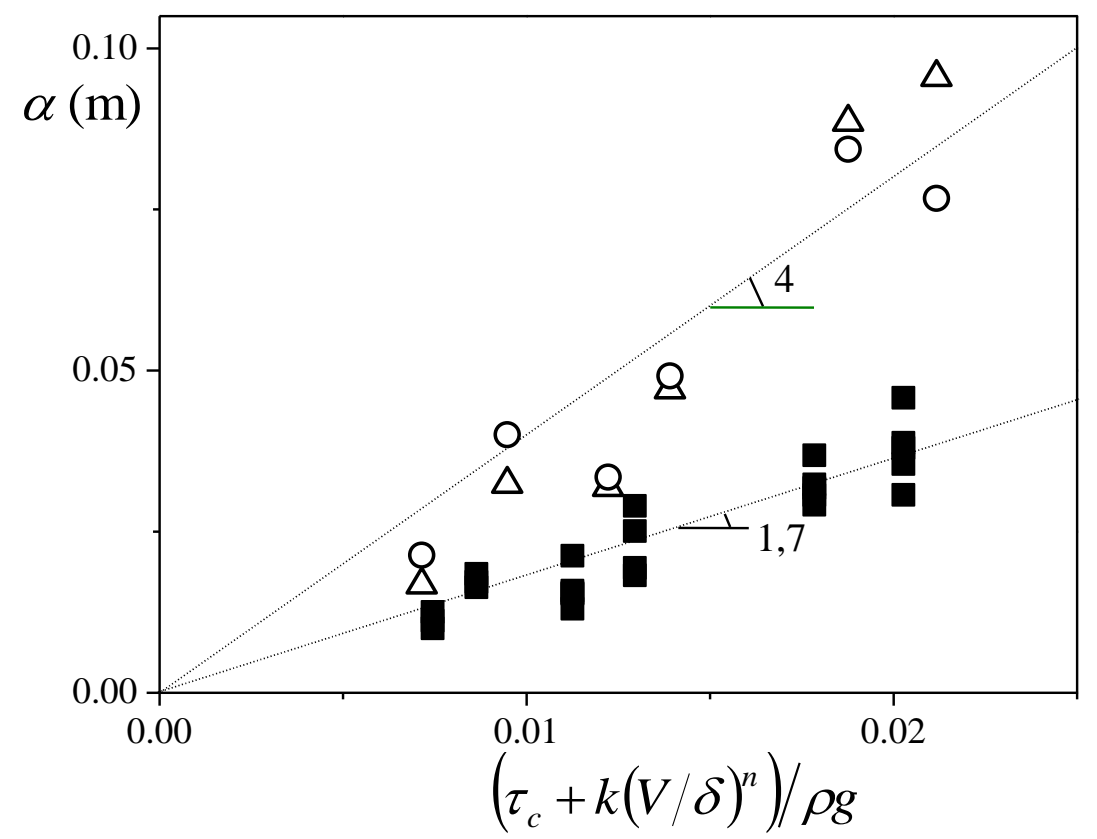

Figure 13: Values of $\alpha$ as a function of the shear stress scaled by gravity for all the tests: (filled squares) our different data for $h_{0} \geq 7 \mathrm{~mm}$; (triangles) $h_{0}=2 \mathrm{~mm}$ and $h_{1}=7 \mathrm{~mm}$, (circles) $h_{0}=2 \mathrm{~mm}$ and $h_{1}=5 \mathrm{~mm}$.

\subsection{Force}

According to the flow scheme presented above we could expect that the force essentially would correspond to the force resisting the gliding of the heap above the layer at rest in the channel, so that it would simply write

$$
F=\tau L D \cos \theta
$$

where $D$ is the channel width and $\theta$ is the angle of inclination of the shear-band. In Figure 14 , in which we plotted various data, we see that this theory is not valid: the force is significantly larger (by a factor between 2 and 5) than the value given by (8).

It is nevertheless remarkable that all the force data rescaled by the shear stress fall along a master curve as a function of the length of the shear-band (see Figure 14). Remarkably the master curve is valid for both regimes (for any $h_{0}$ value). Finally these data are rather well represented (within $25 \%$ ) by the following equation:

$$
\frac{F}{\tau D}=0.03+1.5 L
$$

This suggests that the work supplied by the blade displacement ( $F d x$ for an elementary displacement) induces a viscous dissipation with a mean stress of the order of $\tau$ in a volume larger, say by a factor $\chi$, than the shear-band volume. Indeed under these conditions we get $F d x=\chi e L D \tau \gamma$, in which $\gamma \approx d x / e$ and $e$ the thickness of the shear-band, from which we 
deduce $F=\chi L D \tau$. This could be justified by the fact that even if we essentially observe, in the velocity fields obtained by PIV, a shear-band where most of the shear allowing the heap displacement tends to be localized, some significant amount of fluid inside the heap is progressively deformed in its liquid regime which gives rise to an additional viscous dissipation term (with regards to that associated with the shear-band) in the energy balance. Note that an estimate of the potential energy change due to the vertical displacement of fluid in the heap, shows that this vertical motion plays a negligible role in the viscous dissipation.

This result suggests that the force balance leading to (8) is not exact because it does not include normal stresses acting along the shear-band and resulting from the heap weight and the deformation of the fluid around the heap. At this stage we do not see how to estimate simply the corresponding term, but we can remark that it resembles the effect observed for the displacement of an object through a yield stress fluid i.e. the force is the sum of a term due to the flow of a volume of material in its liquid regime around the object, and a force associated with elastic deformations of the material at larger distance [23-24]. This explanation is also consistent with our interpretation of the result concerning the thickness of the coated layer larger than the gap (see $\$ 4.2$ ). At last this seems to provide an explanation for the finite force recorded when $L$ tends to zero (see Figure 14): a small initial displacement will essentially induce a deformation of the fluid in its solid regime, associated with a fast increase of the force, up to a point where the liquid regime is reached in some part of the fluid; this effect seems to be observable in Figure 10 where the normal force seems to rapidly reach a finite step then increases more slowly.

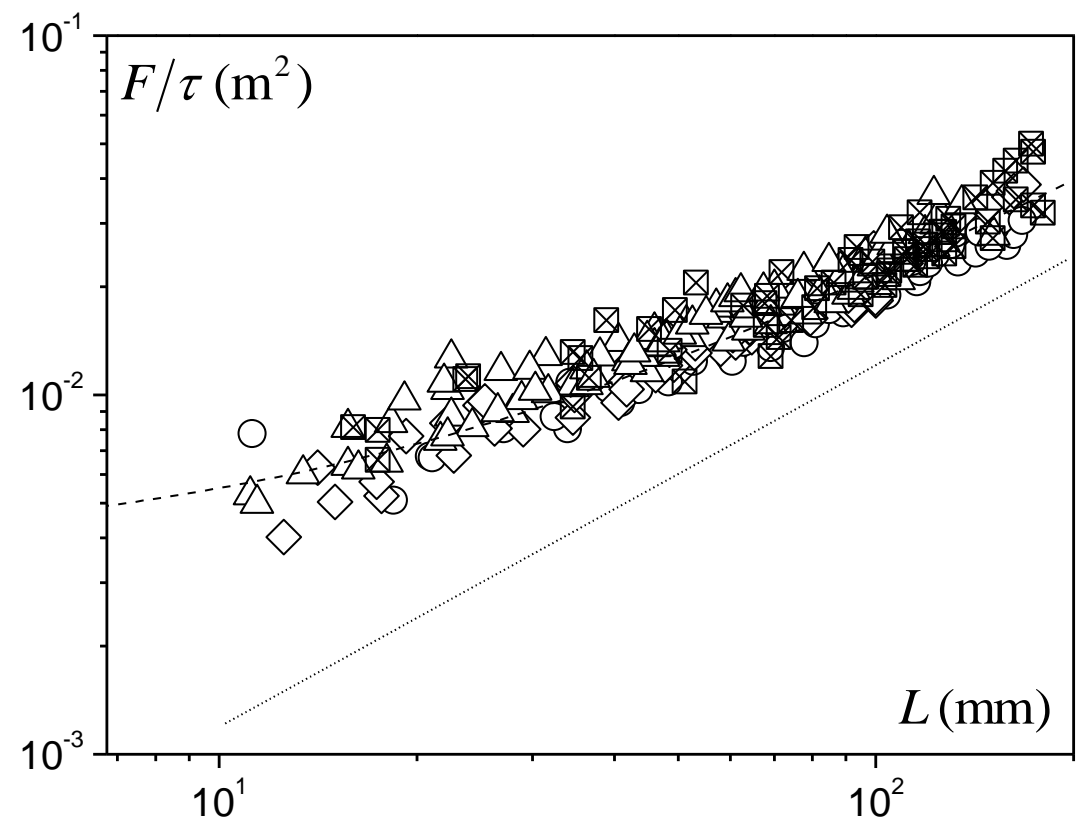

Figure 14: Force scaled by the shear stress (see text) as a function of the length of the shear-band for all our tests (see Table 1) at velocity $V=50 \mathrm{mms}^{-1}: \tau_{c}=42 \mathrm{~Pa}$ (circles), $\tau_{c}=49 \mathrm{~Pa}$ (diamonds), $\tau_{c}=98 \mathrm{~Pa}$ (triangles). Data corresponding to a velocity $V=1 \mathrm{mms}^{-1}$ and $\tau_{c}=98 \mathrm{~Pa}$ are represented by crossed squares. The dotted line is equation (8) with $\theta=0$, the dashed line is equation (9). 


\section{Conclusion}

Our experiments show the original characteristics of the blade-coating of yield stress fluids. First of all the coated layer has a uniform thickness slightly larger than the blade height, which strongly contrasts with the current observation with simple liquids (i.e. significantly smaller thickness). This effect is likely due to the elasticity of the fluid in the solid regime: the scraping of the material by the blade induces some slight elastic squeezing of the bottom layer of the material (still in its solid regime) which then gets back to its initial shape just behind the blade.

A second characteristic of the blade coating of a yield stress fluid is the accumulation of material (heap) at the front of the blade: as a result of the uniform coated thickness behind the blade the volume of this heap grows with the blade displacement. It appears that its shape is close to that resulting from a slump of yield stress fluid submitted to a uniform shear stress at the bottom.

A third aspect is that the fluid is mainly sheared along a layer (i.e. liquid layer) of thickness almost independent of the fluid yield stress, blade height, and velocity. This effect is reminiscent of the flow characteristics recently observed with yield stress fluids under different flow conditions involving the displacement of a mass of fluid relatively to another one (flow through a wedge [15], motion of a plate through a bath of fluid [17]), flow through a larger cavity [19]): the formation of a kind of shear band of almost constant thickness and in which the shear rate is approximately homogeneous. This suggest an origin for the two different regimes distinguished depending on the relative values of the blade height and this sheared thickness: when the blade height is larger than the liquid layer thickness the flow characteristics are independent of the blade height, the thickness of the solid region between the bottom and the liquid layer simply growing with the blade height; when the blade height falls below the liquid thickness the flow characteristics are more complex since the liquid layer cannot keep its thickness at least around the blade.

Future studies could focus on full numerical simulations of this type of flow, or on experiments closer to that encountered when coating mortars of cosmetic pastes, i.e. with a blade inclined close to the horizontal, which likely involves a stronger squeezing flow component.

Ackowledgment: We are grateful to S. Manneville, B. Dollet, P. Saramito, C. Clanet, J. Boujlel, and G. Ovarlez for fruitful discussion in the course of the work.

\section{References}

[1] S.J. Weinstein, K.L. Ruschak, Coating flows. Annu. Rev. Fluid Mech., 36 (2004) 29-53. 
[2] Y. Greener, S. Middleman, Blade-coating of a viscoelastic fluid, Polymer Engineering \& Science, 14 (1974) 791-796.

[3] T.M. Sullivan, S. Middleman, Film thickness in blade coating of viscous and viscoelastic liquids. Journal of Non-Newtonian Fluid Mechanics, 21 (1986) 13-38.

[4] T. Sullivan, S. Middleman, R. Keunings, Use of a finite-element method to interpret rheological effects in blade coating, AIChE Journal, 33 (1987) 2047-2056.

[5] E. Mitsoulis, G. Athanasopoulos, Numerical simulation of blade-over-roll coating forming flows, Computer Methods in Materials Science, 10 (2010) 214-224.

[6] T.C. Hsu, M. Malone, R.L. Laurence, S. Middleman, Separating forces in blade coating of viscous and viscoelastic liquids. Journal of Non-Newtonian Fluid Mechanics, 18 (1985) 273294.

[7] C.J. Guzy, B.G. Higgins, Viscous pressure across the nip of a blade coater and its effect on the final coated film thickness. In Coating Conference Proceedings (pp. 63-71), Atlanta TAPPI Press (1982)

[8] E. Mitsoulis, Numerical simulation of viscoelastic effects in blade-over-roll coating forming flows. Computer Methods in Materials Science, 10 (2010)156-166.

[9] F. Davard, D. Dupuis, Flow visualisation experiments in a blade coating process. Journal of Non-Newtonian Fluid Mechanics, 93 (2000) 17-28.

[10] M. Maillard, J. Boujlel, P. Coussot, Flow characteristics around a plate withdrawn from a bath of yield stress fluid, Journal of Non-Newtonian Fluid Mechanics, 220 (2015) 33-43.

[11] M. Maillard, J. Boujlel, and P. Coussot, Solid-solid transition in Landau-Levich flow with soft-jammed systems. Physical Review Letters, 112 (2014) 068304.

[12] S. Sofou, E. Mitsoulis, Roll-over-web coating of pseudoplastic and viscoplastic sheets using the lubrication approximation. Journal of plastic film and sheeting, 21 (2005) 307-333.

[13] I.J. Hewitt, N.J. Balmforth, Viscoplastic lubrication theory with application to bearings and the washboard instability of a planing plate, Journal of Non-Newtonian Fluid Mechanics 169-170 (2012) 74-90

[14] A. Deblais, R. Harich, D. Bonn, A. Colin, and H. Kellay, Spreading of an Oil-in-Water Emulsion on a Glass Plate: Phase Inversion and Pattern Formation, Langmuir 31 (2015) 5971-5981

[15] D.D. Pelot, N. Klep, A.L. Yarin, Spreading of Carbopol gels, Rheol. Acta, 55 (2016) 279-291

[16] A-T. Akono, P.M. Reis, F-J. Ulm, Scratching as a Fracture Process: From Butter to Steel, PRL 106 (2011) 204302

[17] J. Boujlel, M. Maillard, A. Lindner, G. Ovarlez, X. Chateau, P. Coussot, Boundary layer in pastes-Displacement of a long object through a yield stress fluid. Journal of Rheology, 56 (2012) 1083-1108.

[18] Y. Rahmani, M. Habibi, A. Javadi, D. Bonn, Coiling of yield stress fluids, Phys. Review E, 83 (2011), 056327

[19] T. Chevalier, S. Rodts, X. Chateau, J. Boujlel, M. Maillard, P. Coussot, Boundary layer (shear-band) in frustrated viscoplastic flows. EPL, 102 (2013) 48102. 
[20] M. Maillard, J. Bleyer, A.L. Andrieux, J. Boujlel, P. Coussot, Dip-coating of yield stress fluids, Physics of Fluids, 28 (2016) 053102

[21] P. Coussot, S. Proust, C. Ancey, Rheological interpretation of deposits of yield stress fluids. Journal of Non-Newtonian Fluid Mechanics, 66 (1996) 55-70.

[22] N. Roussel, P. Coussot, «Fifty-cent rheometer" for yield stress measurements: from slump to spreading flow. Journal of Rheology, 49 (2005) 705-718.

[23] J. Boujlel, P. Coussot, Measuring yield stress: a new, practical, and precise technique derived from detailed penetrometry analysis, Rheol. Acta, 51 (2012) 867-882

[24] I. Maimouni, J. Goyon, E. Lac, T. Pringuey, J. Boujlel, X. Chateau, P. Coussot, Rayleigh-Taylor instability in elastoplastic solids: a local, catastrophic process, Phys. Rev. Lett., 116 (2016) 154502 